\title{
Prestaciones para la conciliación de la vida laboral y familiar: una revisión de su diseño e impacto en los países de la OCDE
}

\section{Peru Domínguez-Olabide}

SIIS Servicio de Información e Investigación Social

pdominguez@siis.net

Familia-politikak gizarte-babeserako sistema gizarte-inbertsioaren paradigmara egokitzeko funtsezkoak dira. Politika horien esparruan, kontziliaziorako prestazioak funtsezko osagai gisa aintzatetsi daitezke. Familia-bizitzan eta lan-merkatuan emakumeen eta gizonen arteko partaidetza orekatua sustatzera bideratzen diren heinean, enpleguan ematen den aukera-berdintasun ezari aurre egiteko, genero-rol eta -estereotipo tradizionalak hausteko, eta haurren arreta- eta zaintza-beharrak asetzeko tresna gisa aurkezten dira. Lan honek prestazio horiekiko lehen hurbilketa bat proposatzen du. Ariketa honetan, lehenik eta behin, literatura akademikoa berrikusi da, hizpide diren prestazioak dimentsio ezberdinetan dituzten eraginak ezagutzeko asmotan. Ondoren, prestazio horiek egituratzeko nazioartean leudekeen modu ezberdinak deskribatzen dira.

\section{GAKO-HITZAK:}

Familia-politika, genero-berdintasuna, prestazio ekonomikoak, zerbitzu-ebaluazioa, nazioarteko ikuspegia.
Las políticas de apoyo a las familias con hijos/as resultan fundamentales para la adecuación del sistema de protección social al paradigma de la inversión social. En el marco de estas políticas, las prestaciones de apoyo a la conciliación se constituyen como uno de los pilares fundamentales. Orientadas a impulsar una participación equilibrada entre mujeres y hombres en la vida familiar y en el mercado de trabajo, estas prestaciones se posicionan como herramienta para avanzar en la igualdad de oportunidades en el empleo, romper con los roles y estereotipos tradicionales de género, y cubrir las necesidades de atención y cuidado de los niños/as. Este trabajo realiza una aproximación exploratoria a estas prestaciones. Primero, se realiza una revisión de la literatura académica, presentando las principales evidencias acerca de los impactos de estas prestaciones sobre distintas dimensiones. Seguidamente, se describen las principales formas de estructuración de estas prestaciones en el ámbito internacional.

\section{Palabras Clave:}

Política familiar, conciliación, igualdad de género, prestaciones económicas, evaluación de servicios, panorama internacional. 


\section{Introducción}

El presente artículo pretende ofrecer algunas nociones acerca del estado de la cuestión en cuanto a las prestaciones de apoyo a la conciliación familiar, facilitando a las personas interesadas en la materia una aproximación que combina la perspectiva teórico-académica, con un enfoque comparativo a nivel internacional. El artículo recoge los resultados de un trabajo más amplio realizado por el SIIS Servicio de Información e Investigación Social, para la Dirección de Política Familiar del Departamento de Justicia, Igualdad y Políticas Sociales del Gobierno Vasco, en el que se han analizado también las prestaciones por hijo/a a cargo en los países de la Organización para la Cooperación y el Desarrollo Económico OCDE ${ }^{1}$, así como el impacto de las prestaciones para la conciliación existentes en la Comunidad Autónoma del País Vasco.

En el marco de este trabajo, la concepción considerada del concepto de conciliación estaría en línea con la siguiente definición propuesta por Emakunde (Emakunde, 1996):

La participación equilibrada entre mujeres y hombres en la vida familiar y en el mercado de trabajo, conseguida a través de la reestructuración y reorganización de los sistemas, laboral, educativo y de recursos sociales, con el fin de introducir la igualdad de oportunidades en el empleo, variar los roles y estereotipos tradicionales, y cubrir las necesidades de atención y cuidado a personas dependientes.

El primer punto que cabe matizar en lo relativo a las prestaciones analizadas es el que concierne a las "personas dependientes". En este sentido, se han considerado únicamente aquellas ayudas destinadas a facilitar la atención y el cuidado de los hijos/as. El concepto de atención y cuidado de los hijos/as engloba un sinfín de casuísticas que van más allá de la dependencia que presentan por el hecho de ser niños/as, ya que también engloba las necesidades "extraordinarias" de cuidado, como, por ejemplo, las surgidas por algún tipo de discapacidad o por enfermedades graves. A este respecto, las prestaciones orientadas a las necesidades de atención y cuidados extraordinarios se han dejado de lado.

La familia es una institución que puede presentar multitud de formas. Así, por ejemplo, puede estar compuesta por uno o dos progenitores, que pueden ser o no del mismo sexo. Además, los hijos/as pueden ser biológicos o adoptados, y en algunos casos uno de los progenitores será el padre o la madre biológica, y el otro o la otra, no. En definitiva, dependiendo del contexto sociocultural en cuestión, las familias pueden adoptar infinidad de formas $y$, en algunos casos, esta diversidad tiene su reflejo en las disposiciones de las prestaciones de apoyo a la

${ }^{1}$ Véase en este mismo número el artículo de Zalakain (págs. conciliación familiar. Considerando que el presente trabajo pretende más bien ofrecer una radiografía general, se ha decidido utilizar la familia biparental de hijos/as biológicos como eje del análisis.

El artículo se estructura en dos apartados principales. El primero presenta una breve revisión de la evidencia disponible sobre el impacto de las prestaciones de apoyo a la conciliación familiar en determinados aspectos relacionados con el bienestar infantil, el funcionamiento familiar y la inclusión laboral de los progenitores y, particularmente, de las madres. Para ello, se han analizado estudios y revisiones de la literatura que han intentado determinar las implicaciones que tienen este tipo de prestaciones. En un ejercicio de síntesis, además de dar cuenta de los impactos de las prestaciones de conciliación en las citadas dimensiones, se proporcionan unas pinceladas sobre las principales dinámicas, procesos e interacciones que puedan estar detrás de estos impactos.

El segundo de los apartados presenta algunas conclusiones acerca de los elementos fundamentales de las prestaciones de apoyo a la conciliación familiar vigentes en los países de la OCDE. El análisis se articula de acuerdo a los tres grupos de prestaciones identificadas - prestaciones por maternidad y paternidad, prestaciones por excedencia y prestaciones para la contratación externa de cuidados-, y para cada una de ellas se examinan las principales formas y características que adoptan a nivel internacional.

\section{Evidencia disponible sobre el funcionamiento e impacto de las prestaciones de apoyo a la conciliación familiar}

Bajo el nombre de prestaciones para la conciliación se engloba una amplia diversidad de prestaciones relativamente heterogéneas. Ante la tarea de sistematizar, recopilar y resumir las principales implicaciones para cada tipo de prestación, por un lado, así como la diferencia en el recorrido histórico y en el tratamiento recibido por los distintos tipos de prestación en la literatura especializada, se ha optado por centrar esta revisión en torno a dos categorías de prestación: las prestaciones correspondientes a los permisos que pretenden compensar la reducción en la dedicación laboral de madres o padres (permisos de maternidad, paternidad y excedencias, fundamentalmente) y las prestaciones orientadas a facilitar para la contratación de cuidados externos, bien en centros de atención infantil, bien por parte de profesionales o cuidadores/as a domicilio ${ }^{2}$. Esta categorización de las prestaciones en "prestaciones para la

${ }^{2}$ Respecto a esta última categoría, se ha decidido no hacer mención de los beneficios fiscales debido a la falta de un corpus analítico comparable con las demás prestaciones con las que comparte categoría. 
contratación de cuidados" y "permisos" resulta conveniente, ya que en la literatura especializada se identifican efectos muy dispares para cada una de estas categorías de prestación.

La variedad de prestaciones, contextos y metodologías que se tienen en cuenta en los estudios analizados hace que sea complicado extraer conclusiones universalmente válidas de este apartado. En la literatura revisada se analizan prestaciones con características muy diferentes y que operan en entornos también muy diferentes, por lo que las enseñanzas que de ellas se desprenden pueden estar condicionadas por pequeños matices presentes en su configuración, sin olvidar la importancia del contexto socioeconómico, cultural e institucional en el que se aplican. No obstante, la revisión realizada permite poner de manifiesto algunas conclusiones de carácter generalizable y que pueden contribuir a la reorientación de las políticas de conciliación familiar en otros contextos.

\subsection{La participación de los padres y, especialmente, las madres en el mercado laboral}

Si bien se ha observado un progreso importante en los últimos años, la convergencia entre las tasas de empleo masculinas y las femeninas está lejos de materializarse. En este sentido, puede decirse que, entre otros fines, las prestaciones para la contratación de cuidados y los distintos permisos (maternidad, paternidad o excedencia) pretenden fomentar la participación de la mujer en el mercado laboral.

Antes de proceder a analizar de forma separada los efectos de estas prestaciones en los niveles de empleo de las mujeres, cabe detenerse en las implicaciones que estas prestaciones pueden tener en la demanda laboral por parte de los empleadores. Estas prestaciones incidirían en la demanda de empleo femenino por dos vías principales (Olivetti y Petrongolo, 2017). Por un lado, en la medida en que una parte de los costes de las prestaciones repercute directa o indirectamente en las empresas empleadoras, la demanda de trabajo femenino (especialmente de las mujeres en edad de tener hijos/as) podría verse afectada de forma negativa. Por otro lado, si las medidas facilitan de forma efectiva la continuidad en el empleo y una mayor vinculación femenina al mercado laboral, podrían dar pie a un menor alcance de la discriminación estadística contra la mujer, impulsando por consiguiente la demanda de empleo femenino.

\subsubsection{Prestaciones para la contratación de cuidados}

Las prestaciones dirigidas a apoyar a las familias en la contratación de servicios de cuidado para sus hijos/as tienen por objeto proporcionar sustitutos directos al cuidado parental, facilitando así la participación de los progenitores en el mercado laboral. Si bien es cierto que estas prestaciones no se dirigen específicamente a las madres, el hecho de que sean ellas las que en mayor medida interrumpen su carrera laboral para dedicarse a las labores de cuidado hace que, en gran medida, estas prestaciones puedan entenderse como una herramienta de promoción de la actividad laboral femenina.

La evidencia empírica señala que la posibilidad de acceder a servicios de cuidado en centros de atención infantil es la prestación de apoyo a la conciliación más efectiva para impulsar la incorporación al empleo de las madres. Cabe matizar al respecto, sin embargo, que el aumento en las tasas de participación laboral se produce bajo el requisito de una alta cobertura y disponibilidad, y precios subvencionados (Datta Gupta, Smith y Verner, 2008). La participación pública, ya sea a través de la provisión directa o mediante distintos subsidios y ayudas, es una condición necesaria para el impulso de la participación laboral de la mujer. En caso contrario, confiar exclusivamente en el mercado sería equivalente a un impuesto adicional, y de carácter regresivo, sobre la oferta laboral de las mujeres (Esping-Andersen, 2009). En la medida en que los servicios privados de cuidado infantil de alta calidad estarían fuera del alcance de un gran número de familias, dicho sistema comportaría un fuerte sesgo social, obligando a las madres de renta más baja dejar de lado el empleo para dedicarse a tareas de cuidados (Leitner, 2003). Constatando lo anterior, diversos estudios llevados a cabo a nivel internacional concluyen que mientras el acceso no restringido (en cuanto a precio) a las guarderías conlleva un aumento considerable del empleo femenino, los aumentos de precios tienen un efecto significativamente contrario y conducen a una reducción de la oferta laboral femenina (Simonsen, 2006).

En lo que se refiere a la magnitud del efecto de este tipo de prestaciones en la participación laboral de las mujeres en términos cuantitativos, algunos análisis realizados a nivel de la OCDE apuntan a que, de media, un aumento de medio punto porcentual del gasto en servicios de cuidado infantil (en porcentaje del PIB), se asociaría con un aumento del 1,8\% en el empleo femenino (Olivetti y Petrongolo, 2017).

Sin contradecir estas conclusiones, otros trabajos advierten de que el aumento de la participación laboral se concentra en determinados segmentos de la población femenina. En concreto, diversos estudios apuntan a que las principales beneficiarias de estas prestaciones resultan las madres de menor nivel educativo (Cascio y Whitmore Schanzenbach, 2013; Lefevbre, Merrigan y Verstraete, 2009) y los hogares encabezados por mujeres solas (Gelbach, 2002). Cabe apuntar que estas evidencias provienen de investigaciones realizadas sobre la implantación de distintos programas de expansión gratuita de servicios de cuidado infantil en Canadá y Estados Unidos, y no son necesariamente generalizables a 
otros contextos. Tradicionalmente, ambos países han confiado la provisión de este tipo de prestación al mercado, por lo que las conclusiones resultan consistentes con la existencia del "impuesto regresivo" citado anteriormente. En otras palabras, en dichos contextos la expansión de los servicios de cuidado habría posibilitado a las familias de menor renta poder permitirse económicamente hacer uso de estos recursos, pudiendo reducir su dedicación a las tareas de cuidado e incorporarse en mayor medida al mercado de trabajo.

\subsubsection{Permisos para el cuidado de hijos e hijas}

Las distintas modalidades de los permisos para el cuidado pretenden permitir a los progenitores permanecer vinculados al mercado de trabajo durante las interrupciones temporales de empleo, ayudándoles a conservar el capital humano específico a la empresa o la ocupación. En contraposición a las prestaciones orientadas a la contratación externa de servicios de cuidado, no existe consenso en la literatura especializada acerca del impacto que las prestaciones para la cobertura de permisos y excedencias tienen en el largo plazo sobre la inclusión laboral de las personas con hijos/as.

En conjunto, los distintos autores/as que han analizado las consecuencias económicas de los permisos para el cuidado a nivel internacional señalan que los periodos breves de permiso no imponen grandes costes a los empleadores, y al mismo tiempo confieren beneficios sustanciales a las trabajadoras, lo que se traduciría en un aumento de las tasas de empleo femenino (Olivetti y Petrongolo, 2017; Ruhm, 1998; Thévenon y Solaz, 2013). De esta forma, los periodos breves de permiso permiten a las empresas reasignar temporalmente a personas para que cumplan con las responsabilidades de las empleadas ausentes. Por su parte, las trabajadoras, al facilitárseles el regreso al antiguo puesto tras el nacimiento, tienen mayor capacidad de retener el capital humano acumulado. El permiso facilita el mantenimiento de un vínculo formal con el trabajo y el empleador, por lo que se evita tener que empezar "desde cero" al volver al mercado laboral después del nacimiento o periodos de cuidado.

Sin embargo, los periodos más largos de permisos pueden llegar a provocar efectos negativos en el empleo. La literatura apunta mayoritariamente a que la relación entre el periodo de permiso y el empleo sería "curvilínea" (Boeckmann, Misra y Budig, 2015): los efectos positivos se concentrarían durante los primeros meses, manteniéndose hasta alrededor de los doce meses (Olivetti y Petrongolo, 2017); a partir de esa cota, los efectos empezarían a ser negativos. Por un lado, cuanto más extenso es el periodo de permiso, los costes en los que deberá incurrir el empleador serán mayores. La asignación de los trabajadores de reemplazo puede resultar mucho más difícil, por ejemplo, a causa de una mayor incertidumbre sobre el periodo total de permiso y la posibilidad de que el individuo elija en última instancia no volver al mismo trabajo. Dificultades que pueden verse agravadas, en algunos casos, por las reglamentaciones nacionales sobre el trabajo temporal (Ruhm, 1998; Thévenon y Solaz, 2013). En la otra cara de la moneda, los largos periodos de ausencia laboral pueden generar un deterioro del capital humano y de las habilidades específicas relacionadas con el empleo para las trabajadoras, reduciéndose así su empleabilidad y provocando una tasa inferior de participación laboral (Keck y Saraceno, 2013). Esta dinámica se vería agravada en el caso de las mujeres que tienen hijos/as de forma sucesiva.

De lo anterior puede concluirse que los permisos de una duración moderada (hasta doce meses) están asociados con unos niveles más elevados de empleo femenino, mientras que en el caso de los permisos de larga duración los efectos resultan perniciosos. Si se atiende a la composición de los colectivos beneficiarios de estas prestaciones, la literatura señala que los beneficios sobre el empleo se deben a una mayor participación laboral del segmento de mujeres con menor nivel educativo, teniendo efectos perjudiciales para aquellas mujeres que cuentan con mayor nivel de cualificación (Olivetti y Petrongolo, 2017; Kluve y Schmitz, 2014). Para este último grupo, la importancia de la experiencia laboral y de las habilidades específicas es posiblemente mayor que para las menos cualificadas y, por consiguiente, tienen una mayor penalización, ya que perderían oportunidades de ascenso profesional.

Otro de los elementos que incide en la interacción entre los permisos y la participación laboral femenina es la cuantía monetaria que habitualmente va aparejada a estas prestaciones. Cuanto más generosas son las cuantías, existen mayores incentivos para tomar periodos de permiso más largos, un hecho que, como se ha señalado, parece reducir la participación femenina en el mercado laboral (Cukrowska-Torzewska, 2017). Las tasas de empleo femenino disminuirían con el periodo total del permiso que es remunerado y la tasa media de sustitución asociada a esos permisos (Olivetti y Petrongolo, 2017). Las investigaciones realizadas acerca de las extensiones del periodo de estos permisos, por su parte, señalan que las ampliaciones en el periodo remunerado, en lugar de desplazar al periodo no remunerado, han supuesto un aumento en el tiempo de utilización de los permisos y, por consiguiente, menores niveles de participación en el empleo (Dahl, Løken, Mogstad y Salvanes, 2016).

Algunos autores/as han venido a destacar que cuanto más exigentes resultan los criterios de acceso, al ser menor el número de mujeres que tienen acceso a este tipo de prestaciones, menor es la discriminación estadística que padecen y, por tanto, darían pie a unos niveles mayores de participación en el mercado laboral (Datta Gupta, Smith y Verner, 2008). En cualquier caso, las prestaciones que cubren los permisos y excedencias 
no se dirigen únicamente a las mujeres y su uso por parte de los hombres tiene también efecto sobre la participación laboral de las mujeres. En ese sentido, resulta evidente que en un contexto en el que se haga uso de los permisos de forma una más igualitaria, las posibilidades de la mujer para participar en el mercado laboral aumentan. Una de las herramientas que posibilitan un uso más igualitario de los permisos son las "cuotas paternales". Articuladas de distinta forma dependiendo del contexto, estos mecanismos "castigan” el compromiso desigual de los progenitores en cuanto al cuidado de hijo/a (Datta Gupta, Smith y Verner, 2008; Leitner, 2003). En cambio, la posibilidad de transferir el derecho al permiso entre ambos progenitores sería contraproducente para una mayor utilización de los permisos por parte de los padres. Varios autores/as han venido a interpretar estas evidencias como un problema de “efecto de señal". Esto es, en contextos en los que muy pocos hombres acceden a este tipo de prestaciones, hacer uso de los permisos puede comportar una "señal negativa" y suponer una penalización en su itinerario laboral (Datta Gupta, Smith y Verner, 2008).

\subsubsection{Interacción entre los permisos y las prestaciones para la contratación de cuidados}

La literatura apunta que la interacción entre las categorías de prestación anteriores resulta decisiva a la hora de determinar la efectividad de estas medidas en las tasas de participación laboral femenina. En otras palabras, la composición concreta del paquete de medidas de conciliación que se establece en un país determina la amplitud de sus efectos positivos y negativos en el empleo femenino. En ese sentido, una de las interrelaciones más significativas sería la diferencia de la respuesta del empleo femenino a los permisos en función de la disponibilidad de prestaciones para la contratación de cuidados. Se ha constatado al respecto que los efectos positivos en el empleo femenino de los permisos por maternidad se ven atenuados en los países donde las prestaciones para la contratación de servicios de cuidado infantil son limitadas (Olivetti y Petrongolo, 2017; CukrowskaTorzewska, 2017; Washbrook, Ruhm, Waldfogel y Han, 2011). Estas evidencias sugieren la existencia de sinergias entre ambas categorías de prestación que resultan beneficiosas en términos de una mayor participación laboral femenina.

Sin embargo, a excepción de los países escandinavos, en el contexto de la OCDE los países optan por fundamentar su política en materia de conciliación en una de las dos categorías de prestación (Datta Gupta, 2018). Las categorías de prestación bajo análisis se pueden caracterizar de acuerdo a su naturaleza "familiarista" (Leitner, 2003). Brevemente, las políticas familiaristas tendrían por objeto fortalecer la función de cuidado de la familia, mientras que las políticas no-familiaristas se caracterizarían por la socialización o mercantilización de la función de cuidado de la familia mediante servicios públicos o la provisión de cuidados con base en el mercado (Leitner, 2003). De esta suerte, los permisos se englobarían dentro de las políticas familiaristas, mientras que las prestaciones para la contratación externa de cuidados se englobarían dentro del grupo de las políticas no-familiaristas. Las primeras obstaculizarían la incorporación de la mujer al mercado laboral, ya que el fortalecimiento de la función cuidadora de la familia conlleva de forma implícita a la reproducción del statu quo de las relaciones de género en la provisión de cuidados (Leitner, 2003). Las segundas, por el contrario, ayudarían a debilitar el modelo familiar beckeriano ${ }^{3}$, representando una condición estructural necesaria para una participación más equitativa en el mercado laboral.

A modo de cierre, cabe advertir que los impactos beneficiosos de las políticas de conciliación sobre el empleo podrían estar siendo sobreestimados. La dirección de la causalidad puede ser opuesta y, por tanto, puede que las políticas familiares sean una respuesta a los cambios en las normas sociales respecto al género o la creciente participación laboral de la mujer en el mercado de trabajo (fruto de otros factores externos), y no a la inversa (Olivetti y Petrongolo, 2017).

\subsection{Impacto sobre la brecha salarial entre hombres y mujeres}

Entendida como la diferencia existente entre la remuneración media de los hombres y de las mujeres, la brecha salarial ha sido otra de las cuestiones extensamente analizadas por la literatura en materia de políticas de conciliación. Partiendo desde un punto de vista teórico, puede decirse que se identifican tres dinámicas de carácter económico que afectan a los salarios de las mujeres y que en mayor o menor medida son producto de las medidas de conciliación:

- En primer lugar, en vista de que las políticas de conciliación comportan una serie de costes para las empresas (costes de búsqueda y reemplazo, por ejemplo), se ha sugerido que esos costes adicionales se traducen en una penalización salarial para las mujeres -en la medida en que son ellas las destinatarias o principales beneficiarias de una gran parte de las medidas de conciliación-, debido a que los empleadores les trasladarían dichos costes en forma de salarios relativamente más bajos.

- En segundo lugar, los salarios de las mujeres pueden verse afectados negativamente por la denominada “discriminación estadística”. La disponibilidad de medidas de conciliación puede llevar a los empleadores a creer que las madres

${ }^{3}$ En sus investigaciones sobre el comportamiento de las familias, Becker tomaba como referencia un modelo familiar en el que uno de los integrantes realiza una actividad laboral y el otro se dedica exclusivamente a las tareas de cuidado (Becker, 1981). 
pueden resultar menos productivas, menos fiables y más costosas que otros empleados (Cukrowska-Torzewska, 2017), reduciéndose consecuentemente las retribuciones que están dispuestos a otorgarles.

- En tercer y último lugar, cabe detenerse en los efectos sobre el capital humano. Las prestaciones de conciliación tales como los permisos de larga duración podrían generar un deterioro del capital humano de las beneficiarias, produciéndose una reducción en la experiencia laboral y un deterioro de las habilidades vinculadas al puesto de trabajo, empeorando por consiguiente su posición relativa en el mercado laboral, y afectando finalmente de forma negativa a su nivel retributivo (Keck y Saraceno, 2013)2013.

Estos efectos dependen, en cualquier caso, de la cualificación de las mujeres que se benefician de las prestaciones analizadas. Los efectos negativos en el salario pueden reducirse e incluso invertirse en el caso de que la vinculación al mercado de trabajo y la experiencia laboral sean de gran valor. Según apuntan algunos autores/as, esta casuística es más probable que se produzca en sectores y/o puestos de trabajo donde los costes de búsqueda y reemplazo son importantes, y donde la experiencia laboral es altamente valorada (Olivetti y Petrongolo, 2017). Reforzando esta hipótesis, la evidencia empírica concluye que las madres que regresan a la misma empresa después del periodo de permiso experimentan un crecimiento salarial más rápido que aquellas que cambian de empleador (Waldfogel, 1998). Así, la permanencia en el empleo resultaría clave para que los permisos para la conciliación no penalicen los salarios de las mujeres, lo cual implica que el mantenimiento del capital humano específico a la empresa reduciría los impactos negativos de las prestaciones para la conciliación en los salarios.

Las conclusiones que se extraen de la literatura especializada difieren dependiendo de si se trata de prestaciones para la contratación de cuidados o de las distintas modalidades de permisos para el cuidado, $y$, en el seno de estas últimas, dependerían también de la duración de los permisos. A este respecto, los análisis empíricos apuntan a que los permisos de duración corta y moderada (de hasta seis meses) no generan efectos negativos sobre los salarios. En cambio, los permisos de duración más larga se asocian con efectos significativamente negativos en los salarios, provocando una reducción salarial del orden de entre un 1,5\% y un $3 \%$ (Datta Gupta, Smith y Verner, 2008; Ruhm, 1998; Thévenon y Solaz, 2013).

En lo que respecta a las prestaciones para la contratación de cuidados, la literatura es clara al respecto. Unos mayores niveles de disponibilidad de servicios de cuidado tienden a aumentar la participación laboral de las mujeres, lo que facilita la retención de capital humano específico y se traduce, en último término, en una menor penalización salarial para las madres (ADO2 Investigación y Coaching, 2014; Esping-Andersen, 2009; Datta
Gupta, Smith, y Verner, 2008). En definitiva, podría decirse que cuanto mayor es la capacidad de una prestación para incrementar la participación laboral, menor es el efecto negativo que genera en los salarios. De ahí que, en lo que respecta a la composición, de nuevo sean las mujeres con menores niveles de cualificación las que se ven menos penalizadas en sus salarios, siendo, por tanto, las mujeres de mayor nivel educativo las que ven incrementada su brecha salarial.

Vinculada a la brecha salarial estaría la hipotética profundización en la feminización de sectores que provocarían las prestaciones de ayuda a la conciliación familiar. Según Leitner (2003), la paulatina des-familiarización de los cuidados y el aumento de las tasas de empleo femenino al que han contribuido las prestaciones para la conciliación, en muchos casos, no habrían logrado romper con las relaciones de género que imperan en el ámbito de los cuidados. En realidad, el cuidado formal estaría mayoritariamente provisto por mujeres y las relaciones de género en la provisión de cuidados se verían reforzadas. A este respecto, algunos autores/ as han venido a subrayar que, siendo el principal proveedor de servicios de cuidado, el sector público es el único empleador potencial para una gran fracción de la fuerza de trabajo femenina. Este hecho otorga al sector público una posición de monopsonio a la hora de establecer los salarios. Además, las preferencias sindicales en estos sectores se centrarían más en las mejoras de las condiciones para la conciliación (por ejemplo, permisos más extensos) a expensas de los incrementos salariales. La conjunción de los factores anteriores induciría a unos niveles salariales más modestos en el sector público y, al ser un sector altamente feminizado, ahondaría en la brecha salarial existente (Datta Gupta, Smith y Verner, 2008).

\subsection{Impacto sobre la fecundidad}

De acuerdo con el trabajo seminal sobre la familia de Becker (1981), los factores económicos que determinan la "demanda" de hijos/as en una familia dependen de la renta y los efectos de sustitución. Así, en aquellos contextos en los que se carezca de medidas de política familiar, debido a que los costes de tener un hijo/a aumentarían con el potencial de ingresos de la madre, cabe esperar una correlación negativa entre la oferta laboral femenina y la tasa de fecundidad. Las políticas familiares ayudarían a atenuar esta disyuntiva. En el caso de las prestaciones para la conciliación, los permisos para el cuidado reducen la pérdida inmediata de ingresos por tener un hijo/a, y en la medida en que facilitan una mayor vinculación con el mercado laboral, reducen las posibles penalizaciones en el salario a largo plazo (Datta Gupta, Smith y Verner, 2008). Igualmente, las prestaciones para la contratación de cuidados implicarían una reducción en el coste del cuidado de los hijos/as. 
La irrupción de las políticas de conciliación ha supuesto un cuestionamiento del modelo beckeriano clásico, y la relación entre la oferta laboral femenina y la tasa de fecundidad, otrora negativa, se ha visto invertida (Ahn y Mira, 2002). El nuevo contexto que se ha originado a partir de la implantación generalizada de políticas de ayuda a la conciliación ha facilitado a las mujeres compaginar un empleo con el cuidado de sus hijos/as, y este hecho parece ser favorable para las tasas de fecundidad.

Por un lado, en lo que respecta a las prestaciones para la contratación de cuidados, los resultados apuntan a que el gasto en estas prestaciones tiene una correlación significativamente positiva con la tasa de fecundidad (Datta Gupta, 2018). A nivel de la $O C D E$, se estima que un punto porcentual adicional de gasto en relación al PIB está asociado con 0,2 hijos/as adicionales por mujer (Shim, 2014). Por otro lado, en cuanto a los distintos permisos para el cuidado, la evidencia arroja resultados inesperados. Los datos disponibles indican que la relación entre las tasas de fecundidad y la duración de los permisos es significativa, estando ambas positivamente correlacionadas (Ahn y Mira, 2002; Shim, 2014).

De esta suerte, considerando las prestaciones de forma agregada, la literatura apunta a que las ayudas de apoyo a la conciliación familiar tienen efectos positivos sobre la fecundidad (Shim, 2014; Lalive, 2009; Adema, Ali y Thévenon, 2014; Raute, 2019; Thévenon y Gauthier, 2011). En este punto cabe hacer una mención al proceso de “desmaternalización” al que hacen referencia algunos autores/as. Concretamente, concierne a la autonomía de las madres con respecto a la obligación de atender a las personas dependientes (Mathieu, 2013). Mediante ello, se subraya que lo importante no es solo la transferencia de cuidado fuera de la unidad familiar (“des-familiarización”), sino más bien aligerar la carga de cuidados que descansa de forma específica sobre las madres. Incluso cuando el trabajo de cuidado está muy familiarizado, puede "desmaternalizarse" si es ejecutado por alguien que no sea la madre. El concepto de “desmaternalización” puede ofrecer algunas pistas acerca de la relación positiva entre algunas prestaciones para la conciliación y las tasas de fecundidad. En ese sentido, las prestaciones por paternidad y las prestaciones para la contratación de cuidados permiten transferir las tareas de cuidado de la madre a otra instancia, ya sea al padre o al personal de los distintos servicios de cuidado. La asociación positiva existente entre el uso de la prestación por paternidad y el aumento de segundos y terceros nacimientos constatado en algunas investigaciones vendría a fortalecer la hipótesis anterior (Mathieu, 2013).

Otra condición necesaria para que las políticas de conciliación puedan tener un efecto positivo en las tasas de fecundidad tiene que ver con la aceptación social de la des-familiarización y des-maternalización de los cuidados. En algunos contextos, las actitudes desfavorables hacia el cuidado en el exterior del ámbito familiar reducirían la capacidad de influencia de estas prestaciones en las tasas de fecundidad (Mathieu, 2013). Asimismo, en ciertos casos, el efecto de las políticas familiares sobre la fecundidad podría ser únicamente temporal. Es decir, produciría un adelanto del momento en el que los progenitores deciden tener un hijo/a, en la línea de lo que sucede con determinadas prestaciones por hijo/a a cargo. Cuando las familias sospechan que las prestaciones solo estarán disponibles por un periodo limitado de tiempo, el efecto temporal puede llegar a ser particularmente importante (Lattimore y Pobke, 2008).

\subsection{Impacto sobre la salud y bienestar infantil}

Son cada vez más numerosos y concluyentes los estudios que ligan los primeros años de vida y la intervención precoz con el potencial de desarrollo a lo largo de toda la vida, bajo un paradigma común que cabe englobar en el concepto genérico de los "años fundamentales" (foundation years), en el sentido de que se trata de la fase en la que se colocan los fundamentos de toda la trayectoria vital posterior.

Este énfasis en los años fundamentales se basa tanto en los avances de la sociología y de la pedagogía como, sobre todo, en los resultados que arrojan los estudios neurológicos. Los avances en la investigación neurocientífica están poniendo de manifiesto que las interacciones tempranas del niño con otras personas, máxime con la familia y los cuidadores, establecen las pautas de las conexiones neuronales y los equilibrios químicos que influyen profundamente en lo que nos convertiremos, en lo que seremos capaces de hacer y en cómo reaccionaremos ante el mundo que nos rodea. Todos los aspectos del capital humano adulto, desde las aptitudes de la fuerza de trabajo hasta una conducta lícita y cooperativa, se basan en las capacidades que se desarrollan durante la infancia, empezando por el nacimiento (Adamson, 2008). Así, unas relaciones afectuosas, estables, seguras, estimulantes y gratificantes con la familia y los cuidadores durante los primeros meses y años de vida son esenciales para casi todos los aspectos del desarrollo de un niño.

En relación con lo anterior, como explica Heckman (Heckman, 2006), a la hora de diseñar políticas sociales sólidas dirigidas a la primera infancia habría que prestar especial atención sobre cuatro nociones básicas. Primero, que la arquitectura del cerebro y el proceso de formación de aptitudes se ven influidos por la interacción entre la genética y la experiencia individual. Segundo, que el dominio de aptitudes esenciales para el éxito económico y el desarrollo de sus vías neuronales subyacentes siguen unas normas jerárquicas. Los logros posteriores se sustentan en bases que se establecen con anterioridad. Tercero, que las competencias cognitivas, lingüísticas, 
sociales y emocionales son interdependientes; todas se ven poderosamente influidas por las experiencias del niño en desarrollo y todas contribuyen al éxito en la sociedad en su conjunto. $Y$ cuarto, que pese a la adaptación continúa a lo largo de la vida, las capacidades humanas se forman en una secuencia previsible de periodos sensibles, durante los cuales el desarrollo de circuitos neuronales específicos y las conductas que median son fundamentalmente plásticas y, por tanto, receptivas al máximo a las influencias del entorno.

Se podría decir que la neurociencia y la psicología experimental han demostrado que los primeros años de edad son críticos para el aprendizaje, tanto desde el punto de vista cognitivo como no cognitivo. El aprendizaje sería un proceso dinámico en el que los aprendizajes precoces refuerzan los aprendizajes posteriores, de ahí que algunos autores consideren que las intervenciones resultan tanto más rentables o eficientes (en cuanto a coste) cuanto antes se desarrollen. De acuerdo con un artículo ampliamente citado del propio Heckman (Heckman, Grunewald y Reynolds, 2006), mientras que los programas desarrollados durante la etapa prescolar obtienen una ratio de retorno superior a uno (el beneficio es superior al gasto), los programas aplicados en la escuela y en la formación profesional no alcanzan ese nivel. De ello se desprende que la compensación tardía de un contexto familiar adverso resulta muy costosa.

Por tanto, el periodo prenatal y el posterior al nacimiento son de suma importancia para el desarrollo cerebral del hijo/a. La evidencia científica señala que el desarrollo del cerebro es muy vulnerable a la influencia del entorno y que dicha influencia tiene efectos a largo plazo. Los riesgos son manifiestos: un entorno adverso puede comprometer la función cerebral y el desarrollo general de un niño/a pequeño, exponiéndolo a un mayor riesgo de desarrollar una variedad de dificultades cognitivas, de comportamiento y físicas. En la literatura se identifican los siguientes factores de riesgo como condicionantes negativos para el desarrollo temprano: atención prenatal inadecuada y progenitores aislados (hogares monoparentales, reducción de apoyo familiar, etc.), así como servicios de atención infantil deficientes o de mala calidad, pobreza o atención parental insuficiente, entre otros (Carnegie Task Force on Meeting The Needs of Young Children, 1994).

Ahora bien, dejando de lado los riesgos, las oportunidades también resultan notorias: un entorno favorecedor y estimulante tiene una gran potencialidad para promover el aprendizaje y evitar todo tipo de daños. Los expertos apuntan a la existencia de una serie de "factores de protección" que ayudarían a crear un entorno favorecedor para el niño/a, entre los que se encontrarían las prácticas de cuidado y atención positivas y adecuadas (Carnegie Task Force on Meeting The Needs of Young Children, 1994).
Esta reflexión se relaciona de forma directa con la importancia de los cuidados prestados en la infancia, y especialmente en la primera infancia, y en las fórmulas y mecanismos utilizados para hacer posible la conciliación de la vida familiar y laboral. Las medidas dirigidas a la conciliación familiar favorecen el bienestar infantil a través de distintas vías (Datta Gupta, Smith y Verner, 2008). Por ejemplo, al facilitar el itinerario laboral de las mujeres, contribuyen a que estas sean económicamente más independientes, reduciendo así los niveles de pobreza infantil. Otra de las cuestiones que se plantean en este sentido es la oportunidad que algunas medidas (por ejemplo, la prestación por excedencia o la prestación por paternidad) otorgan a los padres para ser cuidadores activos y adoptar nuevas funciones dentro de la familia.

En función de las distintas etapas de la infancia, las prestaciones de apoyo a la conciliación incidirían de la siguiente forma:

- Durante los meses iniciales después del nacimiento, las prestaciones por maternidad y paternidad son considerados altamente beneficiosos para la salud y bienestar de los niños/as (Datta Gupta, Smith y Verner, 2008; Ruhm, 2000; Waldfogel, 2004). Mediante este tipo de prestaciones, se facilita una atención parental suficiente durante este periodo, crucial para la lactancia materna y para crear lazos de por vida entre los progenitores y el hijo/a. Asimismo, gran parte de la evidencia disponible parece apuntar a que el trabajo de las madres fuera de casa es perjudicial para el desarrollo cognitivo de sus hijos/as durante este primer periodo (Ruhm, 2004; Ermisch y Francesconi, 2001; Shonkoff y Phillips, 2000; National Research Council e Institute of Medicine, 2003). Sin embargo, dependiendo del tipo y la calidad de los servicios sustitutivos de cuidado (Waldfogel, 2004), los efectos perniciosos de la incorporación temprana al mercado laboral podrían verse atenuados.

- Cuando los niños/as tienen entre uno y dos años de edad, las conclusiones anteriores varían de forma considerable. La literatura revisada sugiere que el empleo maternal no conlleva efectos adversos para el desarrollo cognitivo de los hijos/as en este periodo, siempre y cuando los servicios sustitutivos de cuidado sean de calidad suficiente. El recurso a estos servicios puede generar efectos adversos en el comportamiento infantil en caso de que la calidad resulte deficiente (Brooks-Gunn, Han y Waldfogel, 2002).

La cuestión de la calidad es esencial. De la misma forma que unos servicios de calidad durante los primeros doce meses pueden neutralizar los efectos negativos de una incorporación precoz de los padres al mercado de trabajo, unos servicios de escasa calidad durante la etapa posterior pueden neutralizar o incluso revertir las ventajas que se atribuyen a la participación, a partir del primer año, en centros de atención 
infantil. La recepción de cuidados infantiles en estas edades se relaciona positivamente con unos mejores resultados, sobre todo entre los niños de entornos socioeconómicos menos favorecidos (Esping-Andersen, 2009) y siempre y cuando los servicios ofrecidos sean de calidad.

- En tercer y último lugar, cuando los niños/as tienen entre tres y cinco años, las conclusiones respecto al empleo maternal se mantienen. A saber: el uso de servicios de atención infantil de calidad no causa efectos perniciosos para el desarrollo cognitivo, sino todo lo contrario, pero sí efectos adversos en el comportamiento y en el rendimiento académico en caso de hacer uso de servicios de cuidado de calidad deficiente (Brooks-Gunn, Han y Waldfogel, 2002). Adicionalmente, el uso de servicios de cuidado como sustituto del cuidado familiar reduciría los episodios de disciplina física y violencia doméstica (Waldfogel, 2004).

En resumen, el cuidado por parte de los progenitores resulta importante, especialmente durante el primer año de vida, pero la calidad de los servicios externos de cuidado lo es aún más. Por consiguiente, la mejora de la calidad del cuidado no familiar se presenta como una forma efectiva de avanzar hacia unos mayores niveles de bienestar infantil.

\subsection{Impacto sobre el desarrollo infantil, el desempeño educativo, la movilidad social y la igualdad de oportunidades en la infancia}

Se ha argumentado que las etapas iniciales en la vida del niño/a resultan decisivas para su desarrollo cognitivo, por lo que estos primeros años pueden condicionar, en mayor o menor medida, su desempeño educativo y su conducta en los años venideros. En una primera aproximación, cabe subrayar el impacto directo y significativo que tiene el nivel de recursos económicos de las familias en el desarrollo de los niños/as en el corto, medio y largo plazo. Para explicar dicha interacción, en la literatura se proponen dos hipótesis principales: por un lado, se tiende a pensar que la carencia de ingresos incrementa el estrés parental, perjudicando el desarrollo de los hijos/as; por otro lado, desde una posición que toma como referencia la inversión educativa, se tiende a pensar que la disponibilidad de ingresos permite a las familias proveer a sus hijos e hijas de cuidados de mayor calidad y de una mayor inversión en su educación (Gennetian, Castells y Morris, 2010).

Ya sea a través de los distintos permisos (que permiten mantener la vinculación con el mercado laboral, contribuyendo en algunos casos a la mejora en el nivel de ingresos futuro) o las prestaciones para la contratación de cuidados (que reducen los costes de cuidado de los hijos/as), las prestaciones de ayuda a la conciliación inciden de forma indirecta en el nivel de recursos disponibles de las familias. Sin embargo, más allá de los efectos de renta positivos que puedan generar, estas prestaciones tienen efectos tangibles en lo que se refiere al desempeño educativo y a la conducta futura de los hijos/as.

De acuerdo con la literatura consultada, el impacto de los permisos y excedencias sobre el desempeño educativo infantil es nulo (Datta Gupta, 2018). Para intentar explicar la ausencia de efectos positivos, entre otros argumentos, destaca la importancia de la presencia de servicios de cuidado infantil de alta calidad que sustituyan a la atención parental (Carneiro, Løken y Salvanes, 2015). Otra de las dinámicas que pueden limitar el efecto de las prestaciones por permiso es el hecho de que los progenitores realicen inversiones compensatorias. Es decir, pese a llevar a cabo una actividad laboral, pueden dedicar más tiempo (reduciendo, por ejemplo, el tiempo de ocio) o mayores recursos a los hijos/as, sacrificando otro tipo de actividades (Dustmann y Schönberg, 2012). De forma sintética, siempre y cuando los servicios de cuidado alternativos sean de calidad, las prestaciones por permisos no conllevan beneficios significativos en el ámbito de la educación. Además, los progenitores que optan por participar en el mercado laboral acabarían dedicando un tiempo similar al cuidado de los hijos/as en comparación a aquellas que disfrutan de alguna modalidad de permiso.

Continuando en el ámbito educativo, y centrando el foco sobre las prestaciones para la contratación de cuidados $-\mathrm{y}$ en concreto en los servicios de cuidado en centros de atención infantil-, cabe señalar que, en teoría, deberían producir beneficios sustanciales a largo plazo: en primer lugar, porque la receptividad del cerebro es máxima durante la infancia y, en segundo lugar, porque se ha demostrado que la adquisición de habilidades es un proceso acumulativo (Datta Gupta, 2018). No obstante, los beneficios se concentrarían en segmentos concretos de la población. La evidencia apunta a que, sobre todo, la disponibilidad de servicios de cuidado de alta calidad produciría beneficios cognitivos sustanciales para aquellos niños/as pertenecientes a los segmentos de menores ingresos de la población (Love, Eliason Kisker, Ross et al., 2002; Meyers, 2003).

De hecho, la evidencia acerca de la implantación universal de sistemas de atención infantil no arroja resultados tan rotundos. Una gran parte de los trabajos que analizan las expansiones del sistema público de centros de atención infantil reporta efectos positivos en el ámbito de la educación y en la subsiguiente vida laboral, pero estos efectos estarían principalmente guiados por los beneficios adquiridos por los niños/as procedentes de familias con menor nivel de renta (Simonsen, 2006; Cornelissen, Dustmann, Raute y Schoenberg; Herbst, 2017). No obstante, pese a que los efectos positivos más importantes en materia de desempeño escolar se concentran en los niños/as de origen socioeconómico más humilde, los de niveles socioeconómicos más altos también se benefician 
de acudir a estos centros en lugar de hacer uso de acceder a acuerdos de cuidado más informales. En consecuencia, para los niños/as procedentes de estratos socioeconómicos más altos, la evidencia apunta a que cuando el cuidado en centros de atención infantil sustituye a acuerdos informales, los efectos en materia educativa son positivos. En cambio, si antes de la universalización de los centros estos hacían uso de arreglos de cuidado formales (entre otros, los centros de atención infantil privados) los efectos son imperceptibles (Datta Gupta y Simonsen, 2016). Si bien la disponibilidad universal de centros de atención infantil podría implicar un subsidio innecesario para las familias de renta media y alta, las externalidades positivas que pueden generarse de la mezcla de niños/as de diferentes orígenes socioeconómicos, y el mayor apoyo popular de este sistema en comparación con la provisión focalizada, reforzarían la deseabilidad de la universalidad (Datta Gupta, 2018).

En cuanto a los efectos en la conducta de los niños/as tanto a corto como a largo plazo, la conclusión de mayor relevancia sería que la disponibilidad de centros de atención infantil de alta calidad para los niños/as procedentes de familias de bajos ingresos comporta una mejora en las conductas a largo plazo. Ayudarían a mejorar su motivación y sus habilidades sociales, y lo que es más importante, ayudan a reducir los comportamientos futuros relacionados con el crimen, la delincuencia y las adicciones (Heckman, Moon, Pinto, Savelyev y Yavitz, 2010; Heckman y Carneiro, 2003). De nuevo, se pone en evidencia el elevado retorno social implícito en estos programas.

Por último, en consideración de las conclusiones previas, puede deducirse que las políticas de conciliación entrañan elementos que facilitan la movilidad social. En concreto, como se ha podido observar, la provisión de servicios de cuidado de alta calidad en centros de atención infantil favorece fundamentalmente a los niños/as de las familias con menor nivel de renta, en el ámbito educativo y en su posterior vida laboral, y también alejándolos de comportamientos perjudiciales. Por consiguiente, la provisión de servicios de cuidado de calidad se postula como una herramienta efectiva dentro de la política familiar para facilitar la movilidad social de los niños/as de familias con menor nivel de renta y ayudar a construir una sociedad donde se refuerce el derecho a la igualdad de oportunidades (EspingAndersen, 2004).

\section{La estructuración de las prestaciones de apoyo a la conciliación de los países de la OCDE}

Como se ha señalado en el apartado introductorio, en este epígrafe se describen las principales características de las prestaciones de apoyo a la conciliación familiar en los países de la Organización para la Cooperación y el Desarrollo Económico (OCDE). Dada la disponibilidad de datos, el análisis se ha reducido a 34 países de los 37 que conforman la organización ${ }^{4}$.

Siguiendo en buena medida el esquema del apartado anterior, para la realización del análisis las prestaciones para la conciliación familiar se han dividido en tres grandes categorías, cuyos límites son en cierto modo arbitrarios 5 :

- Prestaciones por maternidad y/o paternidad, directamente vinculadas al nacimiento. La prestación por maternidad hace referencia al periodo de descanso remunerado que se les concede a las madres ocupadas en el mercado de trabajo en el periodo posterior $\mathrm{y} / \mathrm{o}$ anterior al nacimiento. Mediante ella, a las mujeres embarazadas, que han dado a luz recientemente o que están en periodo de lactancia, se les garantiza mantener un nivel adecuado de ingresos. La prestación por paternidad es la prestación equivalente dirigida al padre, pareja o cónyuge, concediéndole el derecho a tomar un periodo de descanso inmediatamente después del nacimiento de un hijo/a, asociado con la provisión de ayuda y apoyo a la madre. En general, aunque no siempre, estas prestaciones se dirigen a las personas que están ocupadas en el mercado de trabajo cuando se produce el embarazo y/o el parto.

- Prestaciones parentales por excedencia para el cuidado de los hijos/as. Las prestaciones que se incluyen en este grupo otorgan el derecho a periodos de cese temporal en el empleo y/o reducción de jornada por cuestiones relacionadas con la atención y el cuidado de los hijos/as, una vez finalizado el periodo anterior de permiso por paternidad / maternidad. Se trata de facilitar un periodo de excedencia laboral - total o parcial, y en cualquier caso temporal-que permite a los progenitores que realizan alguna actividad laboral reducir su jornada laboral para cuidar de sus hijos/as durante un cierto tiempo (normalmente, hasta que los hijos/as alcanzan una edad determinada), dándoles cierto grado de seguridad con respecto al empleo. Como se podrá observar en las descripciones para cada país, se distinguen principalmente dos modalidades para esta prestación:

- Por un lado, están las prestaciones por excedencia que no llevan aparejada ningún tipo de remuneración monetaria, es decir, únicamente reconocen el derecho de ausencia temporal del puesto de trabajo.

- Por otro lado, están las prestaciones que más

\footnotetext{
4 Se ha prescindido de Turquía, Colombia y México.
}

${ }^{5}$ De hecho, en algunos países no resulta posible diferenciar entre los permisos por maternidad y paternidad y las excedencias, si bien en la mayor parte de los países la diferenciación es clara si se atiende a criterios tales como la edad de los niños/as, la duración y cuantía de la prestación, las condiciones de transferibilidad, los requisitos de acceso, etc. 
allá de reconocer el derecho a la ausencia temporal, incluyen una ayuda económica para una parte o la totalidad del periodo de excedencia.

- Prestaciones para la contratación de cuidados y sistemas de acceso a los servicios de atención infantil. En este grupo se incluyen todo tipo de ayudas públicas dirigidas a financiar el acceso o la contratación de servicios de cuidado por parte de las familias con hijos/as. En función de su naturaleza, estas prestaciones para la contratación de cuidados se han clasificado en tres subgrupos distintos: acceso a los sistemas públicos de atención infantil, prestaciones económicas para la contratación de servicios de cuidado infantil (en centro o a domicilio) y beneficios fiscales para estos mismos fines.

\subsection{Prestación por maternidad y paternidad}

Antes de proceder, debe señalarse que algunos países - Australia, Dinamarca, Noruega o Sueciano cuentan con una prestación por maternidad/ paternidad en sentido estricto. Disponen de un mecanismo que estaría a caballo entre las prestaciones por excedencia y la prestación por maternidad/paternidad. Por tanto, en algunos países existirían límites difusos entre la prestación por maternidad/paternidad y la prestación parental o por excedencia, lo cual implica que su consideración aislada en una de las dos categorías pueda ofrecer una imagen relativamente distorsionada de las prestaciones.

Otra de las cuestiones previas que cabe destacar se refiere a la consideración del género de las personas

Tabla 1. Paquete de prestaciones para la conciliación familiar articulado en cada país*

\begin{tabular}{|c|c|c|c|c|c|c|}
\hline & $\begin{array}{l}\text { Prestaciones } \\
\text { maternidad }\end{array}$ & $\begin{array}{l}\text { Prestaciones } \\
\text { paternidad }\end{array}$ & $\begin{array}{l}\text { Prestaciones } \\
\text { excedencia }\end{array}$ & $\begin{array}{l}\text { Limitaciones en el } \\
\text { coste de los servicios } \\
\text { de cuidados }\end{array}$ & $\begin{array}{l}\text { Prestaciones directas } \\
\text { contratación de } \\
\text { servicios }\end{array}$ & $\begin{array}{l}\text { Beneficios fiscales } \\
\text { para contratación de } \\
\text { servicios }\end{array}$ \\
\hline \multicolumn{7}{|l|}{ Corea del Sur } \\
\hline \multicolumn{7}{|l|}{ Francia } \\
\hline \multicolumn{7}{|l|}{ Eslovenia } \\
\hline \multicolumn{7}{|l|}{ Finlandia } \\
\hline \multicolumn{7}{|l|}{ Bélgica } \\
\hline \multicolumn{7}{|l|}{ Chequia } \\
\hline \multicolumn{7}{|l|}{ Estonia } \\
\hline \multicolumn{7}{|l|}{ Irlanda } \\
\hline \multicolumn{7}{|l|}{ Noruega } \\
\hline \multicolumn{7}{|l|}{ Reino Unido } \\
\hline \multicolumn{7}{|l|}{ Italia } \\
\hline \multicolumn{7}{|l|}{ Luxemburgo } \\
\hline \multicolumn{7}{|l|}{ Lituania } \\
\hline \multicolumn{7}{|l|}{ Países Bajos } \\
\hline \multicolumn{7}{|l|}{ Alemania } \\
\hline \multicolumn{7}{|l|}{ Austria } \\
\hline \multicolumn{7}{|l|}{ Canadá } \\
\hline \multicolumn{7}{|l|}{ Eslovaquia } \\
\hline \multicolumn{7}{|l|}{ España } \\
\hline \multicolumn{7}{|l|}{ Hungría } \\
\hline \multicolumn{7}{|l|}{ Letonia } \\
\hline \multicolumn{7}{|l|}{ Nueva Zelanda } \\
\hline \multicolumn{7}{|l|}{ Portugal } \\
\hline \multicolumn{7}{|l|}{ Chile } \\
\hline \multicolumn{7}{|l|}{ Dinamarca } \\
\hline \multicolumn{7}{|l|}{ Grecia } \\
\hline \multicolumn{7}{|l|}{ Islandia } \\
\hline \multicolumn{7}{|l|}{ Israel } \\
\hline Japón & & & & & & \\
\hline Polonia & & & & & & \\
\hline Suecia & & & & & & \\
\hline Australia & & & & & & \\
\hline Suiza & & & & & & \\
\hline Estados Unidos & & & & & & \\
\hline
\end{tabular}

* Los permisos por excedencia no remunerados que se compaginan con prestaciones que permiten percibir ingresos durante el periodo de excedencia se consideran en este grupo (Eslovaquia, Estonia, Francia, Letonia).

Fuente: Elaboración propia. 
perceptoras. Algunos países - Islandia, Nueva Zelanda o Israel, por ejemplo- no realizan distinción alguna entre la prestación por maternidad y la prestación por paternidad y conciben, a grandes rasgos, una única prestación por parentalidad dirigida a ambos progenitores. En ese marco, se establecen unas semanas obligatorias de prestación para la madre en el periodo posterior al nacimiento por motivos de salud, y en lo restante se otorga libertad absoluta a los progenitores para dividir ese periodo

\subsubsection{Las prestaciones por maternidad}

\section{A. CRiterios de ACCESO}

En una primera aproximación, puede decirse que se distinguen dos grupos de países en función de las condicionalidades de acceso a la prestación:

- Países que establecen la exigencia de tener vigente un contrato laboral y/o haber trabajado, y por ende cotizado, por un tiempo determinado dentro de un periodo predefinido - mayoría de países-. Se exige la participación previa en el mercado laboral como condición para la percepción de la prestación y se aplica, en general, un principio de contributividad en la medida en que la cuantía de la prestación depende de la cuantía de las cotizaciones previamente realizadas.

- Países que reconocen la universalidad de la prestación, en el sentido de que conceden una prestación de este tipo a todas las madres, independientemente de su participación previa en el mercado laboral o de su afiliación al correspondiente sistema de seguridad social -Alemania, Austria y Finlandia-.

En cuanto al primer grupo de países, si bien en algunos no se exigen periodos previos de cotización -Eslovenia, Estonia, Italia, Países Bajos y Polonia-, lo habitual suele ser el establecimiento de estos, aunque las exigencias resultan variables. Por ejemplo, en Corea del Sur se exige haber cotizado en los seis meses anteriores a la solicitud de la prestación. En España, por el contrario, el requisito se limita a seis meses en los últimos siete años. Adicionalmente, algunos países establecen requisitos en cuanto a las cuantías cotizadas Francia, Reino Unido- o de permanencia previa en la misma empresa -Japón, Reino Unido-.

Otra exigencia habitual es la vigencia de un contrato laboral. En este sentido, el derecho a percibir la prestación por parte de las madres en situación de desempleo varía considerablemente entre países. Pueden distinguirse países que permiten acceder a la prestación a las madres en situación de desempleo, siempre y cuando cumplan con los criterios de cotización establecidos, y otros países que niegan la percepción de la prestación a las madres no ocupadas, aunque cumplan el resto de las condiciones de acceso. Asimismo, algunos países no reconocen como beneficiarias a las trabajadoras por cuenta propia.

\section{B. Determinación de las Cuantías}

La práctica totalidad de los países analizados establece un porcentaje de compensación o tasas de remplazo en función del salario y/o cotización anterior al inicio del periodo de prestación ${ }^{6}$. Irlanda representa la excepción, ya que establece una prestación de cuantía fija. Más allá del establecimiento de distintos porcentajes de compensación, la diversidad de mecanismos para el cálculo de la cuantía de la prestación es notable. Por ejemplo, en países como Bélgica y Finlandia el porcentaje de compensación varía dependiendo de la semana de prestación correspondiente (el porcentaje aplicable está sujeto asimismo al nivel de ingresos anuales del hogar en Finlandia). En todo caso, las cuantías se determinan por lo general en función de los periodos previos de cotización, combinándose habitualmente el principio de universalidad y el de contributividad. En 14 de los 25 países analizados las prestaciones cubren el total del salario anterior.

La mayoría de países establecen límites máximos y/o mínimos a la hora de determinar la cuantía de la prestación. Los criterios aplicados en este ámbito son muy heterogéneos: se suceden los países que no establecen ningún tipo de límite para las cuantías con aquellos que únicamente establecen un umbral superior, un umbral inferior y ambos umbrales.

En cuanto al nivel de esos límites, se observan situaciones muy dispares. Países como Reino Unido imponen umbrales máximos relativamente bajos si se comparan con los salarios medios del país $-28,05 \%-$; por el contrario, España, Luxemburgo o Países Bajos establecen cotas máximas que equivalen aproximadamente al doble del salario medio. De forma paralela, para los umbrales mínimos también se repiten los mismos patrones de divergencia.

\section{Periodos de PRestación}

En términos de duración, el promedio se sitúa en 19,26 semanas. Esta media está en todo caso considerablemente distorsionada por el dato del Reino Unido, que concede 52 semanas de prestación. Asimismo, solamente seis países cubren un periodo superior a las 20 semanas.

Una de las cuestiones centrales sería la distribución del periodo de cobro entre el periodo prenatal y postnatal. Pese a que, por lo general, los países conceden prestaciones que cubren ambos periodos, existen algunas excepciones. Algunos - Suiza, Islandia- no contemplan el periodo prenatal, y otros -España, Corea del Sur-dejan en manos de las

\footnotetext{
${ }^{6}$ Existen infinidad de fórmulas para determinar la base del salario al que posteriormente se aplicará el porcentaje.
} 


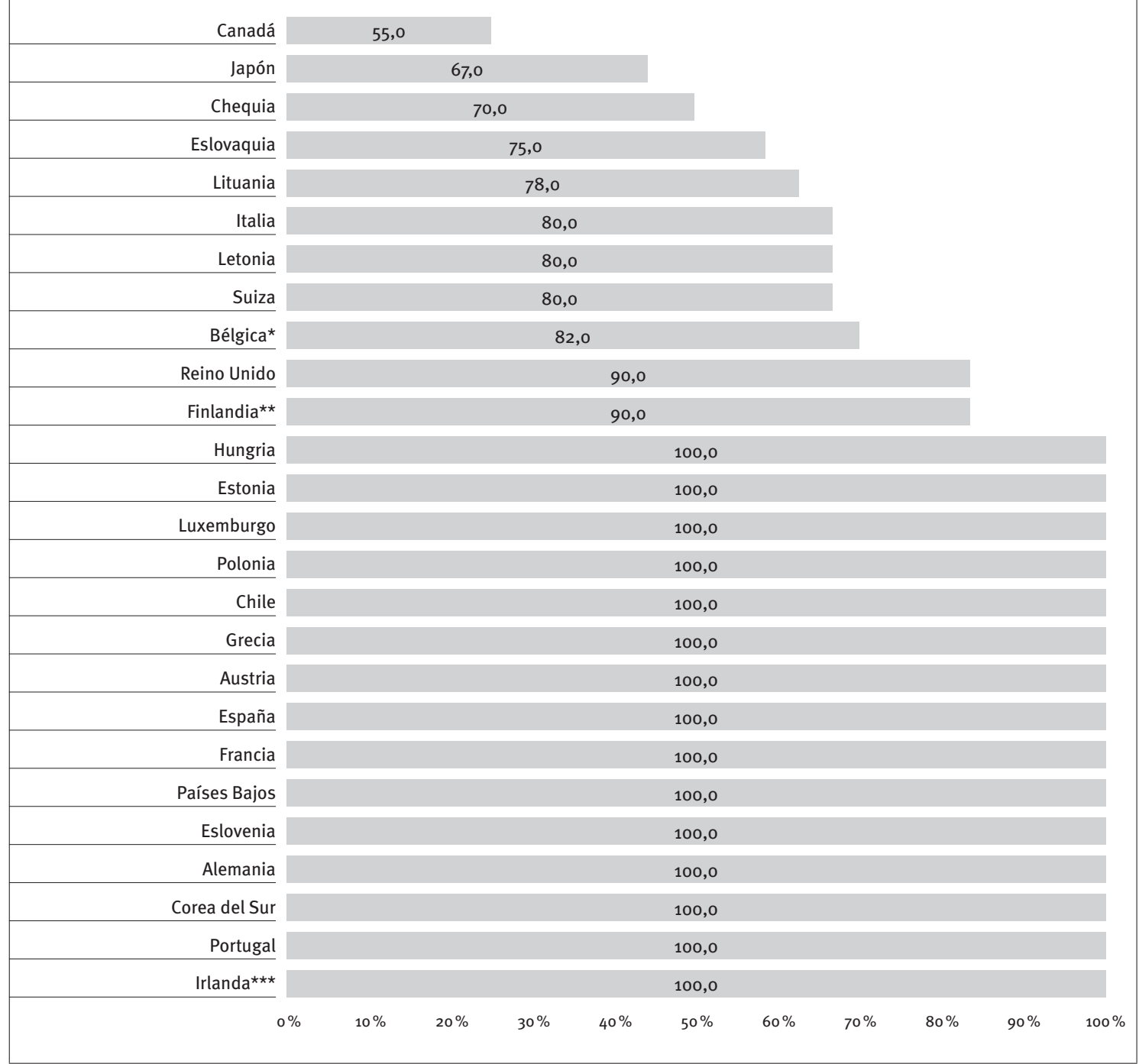

* En Bélgica, a partir de la quinta semana el porcentaje de compensación se reduce al $75 \%$.

** En Finlandia, a partir de la novena semana el porcentaje de compensación se reduce al 70 \%, porcentaje correspondiente al tramo de renta que va de los 10.088,50 euros a los 41.124,11 euros.

*** En Irlanda se otorga una prestación de cuantía fija.

Fuente: Elaboración propia a partir de las fuentes indicadas.

beneficiarias la decisión de si perciben la prestación en dicho periodo. Por lo general el grueso del periodo se reserva para el periodo postnatal.

En aras de una mayor protección de la madre en el periodo de embarazo y lactancia, algunos países establecen periodos de percepción obligatorios?. En un extremo, se sitúan los países que imponen la percepción obligatoria de la prestación para todo el periodo -Alemania, Austria, Grecia, Italia, Luxemburgo-, y, en el otro, aquellos que decretan la voluntariedad de la totalidad del periodo - Lituania y Canadá-. La mayoría de países, sin embargo, combinan periodos voluntarios y obligatorios.

${ }^{7}$ La obligatoriedad, en este caso, se refiere más bien a la obligatoriedad de respetar el periodo de permiso y no incorporarse a la actividad laboral, lo que va acompañado de la percepción de la prestación.
Algunos países incluyen la posibilidad de "transferir" una parte de la prestación por maternidad a la pareja de la madre. Entre los países que contemplan la posibilidad de ceder el título de beneficiario de la prestación al otro progenitor una vez transcurridas algunas semanas de prestación, se observan diferencias sustanciales en cuanto al número de semanas transferibles. El Reino Unido, por su parte, no introduce una "cláusula" para la transmisión del derecho, sino que contempla una prestación separada (shared parental leave), que permite compartir los beneficios de la prestación por maternidad entre ambos progenitores.

\subsubsection{Las prestaciones por paternidad}

De los países analizados, seis de los que disponen de una prestación por maternidad no cuentan 
con una prestación por paternidad -Alemania, Canadá, Chequia, Eslovaquia, Japón y Suiza-. Por el contrario, entre los países que se han dejado de lado en el análisis de la prestación por maternidad por concebir una única prestación por parentalidad para ambos progenitores, Israel y Nueva Zelanda incorporan una prestación separada por paternidad. Ambos se incluyen en este apartado.

\section{A. CRiterios de ACCESO}

En contraste con la prestación por maternidad, solo dos países -Finlandia y Portugal- no establecen un vínculo entre participación laboral y prestación por paternidad. El resto sigue manteniendo también en el caso de los padres requisitos de acceso ligados al mercado de trabajo, principalmente en forma de exigencia de periodos de cotización previos o de vigencia de los contratos laborales.

La principal diferencia de este segundo grupo de países en comparación con la prestación por maternidad es que el número de países que no impone a los padres un periodo mínimo de cotización se extiende de forma considerable. Pese a haberse reducido la muestra de 26 a 22 países, el número de ellos que no impone ningún tipo de exigencia de cotización para el acceso a la prestación aumenta de 8 a 13. A la inversa, si se examina el número de países que conceden el derecho de acceder a la prestación a personas desempleadas y a los trabajadores por cuenta propia, se advierte un drástico descenso. Aparte de reducirse considerablemente el número de países que conceden acceso a estos colectivos, otros lo dificultan con el establecimiento de condiciones más restrictivas para el acceso en comparación con las personas empleadas por cuenta ajena -Francia-.

\section{B. Determinación de LAS CuAntías}

La práctica totalidad de los países establece un porcentaje de compensación en función del salario anterior al inicio del periodo de prestación - existen infinidad de fórmulas para determinar la base del salario al que posteriormente se aplicará el porcentaje-. La excepción la conforman Austria e Irlanda, en los que se concede prestación fija. Nuevamente, la diversidad de los mecanismos de remuneración es considerable. A modo de ejemplo, en Bélgica el porcentaje de compensación respecto al salario varía dependiendo del día de prestación correspondiente. En Israel, dependiendo del día de prestación, se suceden días remunerados y no remunerados. En Finlandia, el porcentaje de compensación aplicable está sujeto al nivel de ingresos anuales del hogar. Con todo, en 12 de los 22 países analizados las prestaciones cubren el total del salario anterior.

Una de las particularidades que presentan las prestaciones por paternidad en algunos países es la responsabilidad de la empresa como agente que otorga las prestaciones. En algunos países -Chile, Corea del Sur, Grecia y Reino Unido ${ }^{8}$ - las empresas están obligadas a otorgarlas de forma exclusiva, y en otros de forma conjunta con los organismos gubernamentales - Bélgica, Luxemburgo, Países Bajos-. En estos últimos, la empresa se encarga de abonar las cantidades durante los primeros días de prestación, mientras que los organismos públicos cogen el testigo durante las semanas siguientes, normalmente por un periodo más largo.

Se establecen, asimismo, límites mínimos y máximos a la hora de determinar las cuantías de la prestación. De nuevo, se entremezclan países que no imponen ningún tipo de límite con otros que únicamente contemplan un umbral superior, inferior, o los que contemplan ambos. Como ocurría con la prestación por maternidad, en relación con el salario medio de cada país las diferencias en el nivel fijado por los límites son considerables.

\section{Periodos de prestación}

El promedio de días de prestación se sitúa en 18,4 días. Se trata de una duración media muy inferior a la establecida para las prestaciones por maternidad. Además, existe una asimetría considerable entre países, mayor probablemente que en el caso de las prestaciones por maternidad. España y Finlandia, con generosos periodos de prestación en términos comparativos - de 16 y casi 8 semanas, respectivamente-, contrastan con otros países que establecen periodos relativamente cortos de unos pocos días -Chile, Grecia, Hungría, Israel, Italia-.

En cuanto al reparto entre el periodo prenatal y postnatal, la prestación se circunscribe en general al periodo postnatal. Algunos países -Grecia, Israel, Lituania y Luxemburgo- establecen que la percepción ha de realizarse en los días inmediatamente posteriores al nacimiento. El resto establece un periodo límite para el disfrute, que va desde las cuatro semanas - Chile y Corea del Sur-, hasta el sexto cumpleaños del hijo/a -Eslovenia-.

Otra línea divisoria entre países se encuentra entre aquellos que obligan a tomar los días correspondientes de forma consecutiva y aquellos permiten dividir la prestación en distintos subperiodos. Entre estos últimos, algunos contemplan la posibilidad de disfrutar de la prestación a tiempo parcial -Eslovenia y España-. Otros establecen ciertos criterios que limitan dichos subperiodos. Por ejemplo, España y Países Bajos establecen que los periodos de prestación habrán de tener una duración mínima de una semana. Finlandia, por su parte, establece un máximo de cuatro bloques para dividir el periodo de prestación.

${ }^{8}$ Pese a ser remunerada directamente por el empleador, posteriormente se les compensa con una rebaja de las cotizaciones sociales. 
Por último, pese a ser mayoritariamente voluntaria, cabe señalar que en algunos países se establece la obligatoriedad de percepción. En algunos casos, la obligatoriedad concierne a todo el periodo de prestación -Italia, Chile-, mientras que en otros casos corresponde a una parte del periodo -España, Portugal-.

\subsection{Prestaciones por excedencia}

A excepción de Chile y Suiza, todos los países de la muestra cuentan con una prestación por excedencia 0 , al menos, reconocen el derecho a la excedencia de las madres y, en algunos, los padres, para el cuidado de los hijos/as. En todo caso, conviene recordar algunos elementos respecto al diseño de estas prestaciones al objeto de poder interpretar adecuadamente la información que se ofrece en este apartado:

- Hay que tener en cuenta que la cobertura de estas situaciones implica en realidad dos derechos: el derecho a abandonar temporal y/o parcialmente el puesto de trabajo y de poder volver a él al terminar el periodo de permiso o excedencia, y el derecho a percibir por parte de la administración una prestación económica para cubrir la pérdida de ingresos derivada de tales permisos o excedencias. Algunos países no reconocen ninguno de estos derechos, otros reconocen únicamente el derecho a la excedencia durante un periodo determinado y otros, además, garantizan una prestación económica durante ese periodo.

- Los límites entre estas prestaciones y las prestaciones por nacimiento o por maternidad y paternidad no siempre tienen un límite claro. En ese sentido, en este epígrafe se incluyen algunos países que cuentan con una prestación que presenta límites difusos entre la prestación por maternidad/paternidad y la prestación por excedencia, y que no han sido por tanto incluidos en el análisis realizado en el apartado anterior.

\subsubsection{Criterios de acceso}

A pesar de que por definición se trata de un periodo de excedencia laboral, no todos los países vinculan esta prestación económica a la interrupción de la actividad laboral, en la medida en que también reconocen el derecho a la prestación a aquellas familias que no realizan ningún tipo de actividad laboral. De esta suerte, algunos países -Alemania, Austria, Chequia, Eslovaquia, Eslovenia, Finlandia, Portugal y Suecia- extienden el derecho a la prestación por excedencia a personas que en el momento de solicitarla no están ocupadas en el mercado laboral. Cabe señalar que todos estos países otorgan cuantías económicas por la prestación por excedencia, de manera que más allá de una excedencia para el cuidado, en estos países las prestaciones se pueden concebir como una ayuda adicional para la atención y el cuidado de los hijos/as.

El resto de países, ateniéndose en mayor medida a la definición de la prestación, establecen criterios de acceso relacionados con la participación en el mercado laboral. Algunos determinan únicamente la necesidad de tener vigente un contrato laboral, sin establecer ningún otro tipo de exigencia en materia de periodos previos de cotización o trabajo y limitando la percepción de la prestación a las personas ocupadas. El resto establece requisitos de cotización o de tiempo de trabajo previos en la empresa actual. En este sentido, los países considerados podrían agruparse en tres grandes grupos:

- Países que únicamente exigen un contrato laboral vigente y no conceden acceso a las personas desempleadas -España, Estonia, Italia, Letonia, Países Bajos y Polonia-.

- Países que establecen periodos mínimos de cotización y no conceden acceso a las personas desempleadas -Australia, Canadá, Corea del Sur, Islandia, Lituania, Luxemburgo, Noruega, Nueva Zelanda-.

- Países que establecen periodos mínimos de cotización y conceden acceso a las personas desempleadas -Dinamarca y Hungría-.

- Países que establecen tiempos de trabajo mínimo en la empresa actual -Bélgica, Estados Unidos, Francia, Grecia, Irlanda, Israel, Japón, Reino Unido-.

Otra característica central corresponde a la titularidad de la prestación. Puede ser de dos tipos:

- Familiar, cuando la elegibilidad se vincula a la situación de ambos progenitores, estableciéndose un periodo concreto para ambos, en el que solo uno puede disfrutar de la excedencia.

- Individual, si a cada uno se le asigna un periodo y tienen la posibilidad de disfrutarlo de forma simultánea.

La muestra de países se encuentra dividida en este aspecto: la mitad de los países analizados consideran la titularidad de la prestación en términos individuales y la otra mitad lo hace en términos familiares.

En relación con la posibilidad de percibir la prestación de forma simultánea por parte de ambos progenitores, por una parte, en algunos países donde la titularidad es familiar, existe la posibilidad de que ambos progenitores perciban la prestación de forma simultánea. No es que solamente exista dicha posibilidad, sino que además puede comportar mayores beneficios tanto en términos monetarios -Alemania, Austria- como en términos de extensión del periodo de prestación -Portugal-. Por el 
contrario, también se observan países en los que se establece la titularidad individual pero que limitan el disfrute conjunto de la prestación. Por ejemplo, Suecia limita el número de días conjuntos elegibles a treinta, acotando su derecho al primer año después del nacimiento del hijo/a. Israel, por su parte, no permite la percepción conjunta.

En lo que se refiere al género de las personas que pueden acogerse a la prestación, ninguno de los países analizados limita el derecho a beneficiarse de esta prestación a los hombres o a las mujeres, pudiendo ambos progenitores, en todos los casos analizados, acceder a la ayuda en las condiciones señaladas previamente. Tampoco se han encontrado casos de países en los que el derecho de acceder a las prestaciones por excedencia esté vinculado a niveles determinados de renta.

\subsubsection{Determinación de las cuantías}

La primera distinción puede realizarse entre los países que ofrecen algún tipo de prestación monetaria y los que no ofrecen ninguna, y se limitan a garantizar el derecho a la excedencia y el posterior retorno al puesto de trabajo.

En cuanto a los segundos, puede decirse que únicamente otorgan el derecho a un cese protegido de la actividad laboral. Sin embargo, desde una perspectiva más amplia, se constata que algunos países -Francia, Estonia y Letonia- tienen vigentes prestaciones complementarias que permiten a las familias obtener ingresos adicionales durante el periodo en el que se acogen al permiso por excedencia. En otros términos, la prestación por excedencia se articularía con otras prestaciones monetarias permitiendo percibir ingresos adicionales durante el periodo de cese de la actividad laboral.

En cuanto a los países que remuneran el periodo de excedencia, una de las distinciones que de entrada resulta posible realizar concierne a la forma de remuneración. La muestra de países se divide entre aquellos que determinan la cuantía sobre la base de un porcentaje fijo de compensación sobre su salario anterior, y aquellos países que establecen una cuantía fija, siendo este último grupo el minoritario -Australia, Bélgica, Chequia, Eslovaquia-. En cuanto a los países que remuneran la excedencia en función del salario anterior, algunos establecen límites máximos para las cuantías, otros umbrales mínimos, y otros establecerían ambos.

En este punto, cabe referirse a la posibilidad de modular la cuantía de la prestación y su duración de acuerdo a la preferencia de las personas beneficiarias de la prestación en algunos países. En ese sentido, se pondría a elección de las familias el poder acceder a otra variedad de prestación, consistente en un periodo más extenso y con unas remuneraciones inferiores -Alemania, Austria, Canadá, Lituania y Noruega-.

\subsubsection{Periodos de prestación}

El primer elemento de divergencia de la muestra se encontraría en la edad de los hijos/as hasta la que se puede continuar con la percepción de la prestación. En una primera observación pueden distinguirse tres grupos de países:

- Países que establecen que el periodo de excedencia ha de tomarse en el primer año de vida del hijo/a. En la práctica, la prestación parental se concebiría como una herramienta que permite extender los periodos de maternidad y paternidad y que se vincula, de forma extensa, al nacimiento y los primeros meses de vida del niño/a -Alemania, Australia, Austria, Canadá, Dinamarca, Estados Unidos, Finlandia, Israel, Japón, Noruega, Nueva Zelanda, Portugal-.

- Países que establecen que el periodo de prestación ha de realizarse entre el segundo y el cuarto año de edad del hijo/a. Podría decirse que en estos casos la prestación parental se erige como una ayuda para facilitar las necesidades de atención y cuidado de los niños/as en la etapa de la primera infancia - Chequia, Eslovaquia, España, Estonia, Francia, Hungría, Lituania, Polonia-.

- Países en los que el periodo de prestación se extiende considerablemente en el tiempo (más de cuatro años). La prestación por excedencia se entendería en estos países como un derecho de excedencia ante cualquier contingencia durante una gran parte de la infancia e, incluso, la adolescencia -Corea del Sur, Eslovenia, Grecia, Irlanda, Islandia, Italia, Letonia, Luxemburgo, Países Bajos, Reino Unido, Suecia-.

La duración de los periodos de excedencia remunerada oscila entre los 48 -Chequia- y los cinco meses -Australia-, siendo el promedio ligeramente superior a 14 meses. En lo que respecta a las excedencias no remuneradas, oscila entre los 36 -España, Francia, Estonia-y los tres meses -Estados Unidos-, con una duración media de alrededor de siete meses y medio. Ambos promedios, en cualquier caso, están muy determinados por las diferentes duraciones de los países analizados.

En último lugar, resulta interesante comentar que en un número considerable de países existe la posibilidad de percibir la prestación a tiempo parcial. Así, las excedencias pueden cubrir tanto el cese total de la actividad laboral como, en algunos casos, la reducción de la jornada laboral durante el periodo establecido -Alemania, Austria, Bélgica, Eslovenia, España, Finlandia, Luxemburgo, Suecia-. Cabe señalar, además, que en algunos países -Bélgica, Luxemburgo, Suecia-, la modalidad a tiempo parcial va aparejada a una extensión proporcional del periodo de prestación. 


\subsection{Prestaciones para la contratación de cuidados}

\subsubsection{Acceso a los sistemas públicos de atención infantil}

El sistema público de atención infantil conforma un complejo sistema en el que, dependiendo del contexto, pueden llegar a confluir instituciones tan diversas como el mercado, la familia, las políticas de educación y las políticas sociales. Por tanto, en vista de las configuraciones institucionales tan dispares que presentan los países analizados, la realización de un análisis comparativo resulta complejo. El análisis se vuelve más complicado si se tiene en consideración que en una parte significativa de los países incluidos en la muestra las competencias relativas a los centros públicos de atención infantil recaen sobre unidades territoriales subestatales. En ese contexto, el análisis que se incluye a continuación se limitará únicamente a ofrecer algunas pinceladas sobre el contenido de las políticas públicas implementadas en los países de la muestra que buscan ayudar a las familias en el acceso a centros de atención infantil.

El primer elemento de interés se vincula con el tramo etario de los hijos/as para el acceso al sistema. La gran mayoría de países establece como periodo de finalización del derecho de acceso el momento en el los niños/as alcanzan la edad de inicio correspondiente a la educación primaria obligatoria, el cual varía de forma notable entre los países analizados. En cuanto a la edad mínima de acceso, existen diferencias notorias:

- Países en los que el periodo contemplado de acceso comienza una vez pasado un breve periodos de tiempo -de entre 6 y 18 mesesdesde el nacimiento -Estonia, Letonia, Irlanda, Dinamarca-.

- Países en los que la edad mínima de acceso se establece a los tres años. Normalmente, las ayudas se materializan mediante la disposición de una red pública de centros para la primera infancia, previendo para el periodo anterior el cuidado en el hogar (mediante la prestación por excedencia) o a través del mercado -Eslovaquia, España, Nueva Zelanda-.

Un segundo elemento de relevancia concierne a la gratuidad de los centros de atención infantil y la determinación del copago que deben asumir las familias. Chile y Letonia son los dos únicos países que desde una edad temprana establecen la gratuidad total en el acceso. En lo que respecta a Letonia, en caso de no haber plazas suficientes en la red pública, deberá garantizarse el acceso a un centro privado de forma gratuita. De forma más limitada, otro grupo de países también contempla la gratuidad de los centros de atención infantil, pero restringiéndolo a los beneficiarios que alcanzan una edad mínima -Chequia, Eslovaquia, España, Israel, Nueva Zelanda, Suecia-. Por otro lado, pese a no decretar la gratuidad de los centros, en la práctica, para las familias que cumplen con una serie de criterios (principalmente, nivel de renta y número de hijos/as) se posibilita el acceso gratuito a los servicios de cuidado en centros de atención infantil en algunos países -Dinamarca, Eslovenia, Finlandia o Japón-.

Los países que no ofrecen una provisión gratuita (o para los tramos de edad para los que no se contempla) existen diversos mecanismos para limitar el gasto realizado por las familias. Por un lado, algunos países establecen un umbral de gasto máximo para las familias. Pueden adoptar la forma de una cuantía fija, una cuantía variable en función de un porcentaje sobre el nivel de ingresos, 0 combinar ambos criterios. Por otro lado, hay países que contemplan la subvención de un porcentaje determinado de los gastos de los centros de atención infantil. Este porcentaje varía principalmente en función del nivel de renta de los progenitores.

\subsubsection{Prestaciones para la contratación de servicios de cuidado infantil}

Consisten en la percepción de prestaciones económicas para el pago de servicios de cuidado en centros de atención infantil o a domicilio por parte de cuidadores/as profesionales. Pueden percibirse tanto de forma directa, mediante la recepción de ayudas económicas por parte de las familias, como indirecta, mediante disposiciones para regular el coste de estos servicios para las familias.

Antes de iniciar la descripción de estas medidas, conviene señalar que la no disponibilidad de prestaciones para la contratación de cuidados no conlleva necesariamente una posición de desventaja en el ámbito de la conciliación. De hecho, entre los países carentes de estas prestaciones se encuentran algunos con sistemas públicos de atención infantil muy desarrollados, como es el caso de Suecia, por lo que antes de extraer ninguna conclusión conviene adoptar un enfoque más estructural, contemplando la articulación de estas prestaciones con los sistemas públicos de atención infantil. En algunos casos, las prestaciones para la contratación de cuidados pueden también convertirse en una de las piedras angulares del sistema público de atención infantil, ya que, entre otros, pueden garantizar el acceso a servicios de cuidado fuera del ámbito familiar a grandes segmentos de la población. El kinderopvangtoeslag neerlandés resulta un ejemplo paradigmático de lo anterior.

Se puede plantear una primera clasificación en función de si las prestaciones se orientan a la contratación de servicios de cuidado en centros o en el hogar. 
3.3.2.1. Prestaciones para la contratación de cuidados en centros

\section{A. Criterios de acceso}

- Diferencias en las edades máximas de los hijos/as. Por un lado, se observan países que contemplan la prestación para distintas etapas de la infancia, pudiéndose acceder a la misma hasta cumplir 11-15 años -Australia, Canadá, Irlanda, Reino Unido, Países Bajos-. Otro grupo de países reservan estas prestaciones para la primera infancia, justamente hasta que los hijos/as cumplan los 3-5 años - Corea del Sur, Eslovaquia, Italia, Nueva Zelanda-.

- Obligatoriedad de realizar algún tipo de actividad laboral o formativa. Pese a la existencia de excepciones, sería la norma general.

- Nivel de ingresos de las familias. Algunos países aplican criterios selectivos a estas ayudas y establecen un umbral de renta a partir del cual no se conceden las prestaciones -Australia, Irlanda, Nueva Zelanda y Noruega-.

- Características sociodemográficas. En algunos países las ayudas se circunscriben a familias que presentan características socioeconómicas concretas, como, por ejemplo, hogares monoparentales o personas jóvenes - Noruega, Nueva Zelanda-.

\section{B. Determinación de LAS aYUdas}

- La cuantía de las prestaciones en la práctica totalidad de los países varía en función de una serie de características familiares. Las variables más habituales serían el nivel de ingresos, la edad de los hijos/as y el número de hijos/as. Igualmente, pero de forma más minoritaria, también se toman en consideración el número de horas dedicadas a la actividad laboral o formativa, el número de horas de cuidado (tiempo completoparcial) de los que hacen uso las familias, o la titularidad (público-privada) de los centros en los que se pretenden contratar los servicios de cuidado.

- Puede decirse que existen dos formas principales para la articulación de las cuantías:

- Países en los que la prestación se otorga en función de un porcentaje sobre los gastos totales de cuidado. Dentro de este grupo, se distinguen algunos países que optan por establecer la gratuidad de los servicios de cuidado para segmentos concretos de la población (en función del nivel de renta), sujeto a algunos límites de utilización.
3.3.2.2. Prestaciones para la contratación de cuidados en el hogar

\section{A. Criterios de acceso}

- La edad máxima de los hijos/as para la elegibilidad de la prestación se reduce considerablemente en comparación con las prestaciones para la contratación de cuidados en centros. Salvo el caso de Luxemburgo -13 años-, el rango máximo de edad para la prestación que establecen la totalidad de países oscila entre los 2-3 y 6 años.

- Aunque por lo general las prestaciones se destinen a la financiación de cuidados en el propio hogar de la persona beneficiaria, existen algunas excepciones. Islandia y Luxemburgo, por ejemplo, disponen de ayudas para el cuidado de los hijos/as en los hogares de familias de terceros.

- Existen diferencias notables en cuanto a los requisitos de acceso más allá de la edad. Mientras que algunos países no imponen ningún requerimiento adicional, la realización de algún tipo de actividad laboral o formativa, los periodos mínimos de residencia en el país, los periodos mínimos de afiliación al sistema de seguridad social o la no recepción de otras prestaciones y ayudas públicas son los principales requerimientos adicionales observados.

\section{DETERMINACIÓN DE LAS AYUDAS}

- Se observa que las cuantías de la prestación varían en función de una serie de características familiares. Sin embargo, el número de características que se toman en consideración son mínimas comparativamente, llegando en algunos casos a establecerse una cuantía fija independientemente de las características familiares. Las principales variables utilizadas son la edad de los hijos/as, el número de hijos/ as, el nivel de renta, las horas totales de cuidado y la utilización de cuidados nocturnos o en días festivos.

- La forma principal para la articulación de estas prestaciones es la dotación de un importe monetario, que oscila significativamente dependiendo del país en cuestión.

\subsubsection{Los beneficios fiscales para la contratación de servicios de cuidado infantil}

El uso de herramientas fiscales para dar apoyo a las familias en la atención y el cuidado de los hijos/ as no sería una herramienta muy extendida. En cuanto al tipo de beneficio fiscal aplicado, adoptan principalmente la forma de desgravaciones fiscales o créditos fiscales. De forma sucinta, mediante las primeras se puede beneficiar de una reducción en la base imponible del impuesto, mientras que a través 
de la segunda se permite reducir el montante de la deuda fiscal correspondiente al contribuyente.

Un primer elemento relevante corresponde al tipo de gasto al que se le puede aplicar el beneficio fiscal:

- Beneficios fiscales para el cuidado en el ámbito domiciliario - Lituania, Suecia, Irlanda-.

- Beneficios fiscales para el cuidado en centros de atención infantil -Corea del Sur, Estonia, Portugal-.

- Beneficios fiscales para el cuidado en el ámbito domiciliario y en centros de atención infantil -Alemania, Bélgica, Canadá, Chequia, Estados unidos, España, Francia, Italia-.

Además de los gastos referidos, algunos países permiten también la aplicación de beneficios fiscales con relación a los gastos realizados en actividades de ocio y tiempo libre -Canadá, Chequia-.

La franja de edad contemplada para la aplicación de los beneficios difiere sustancialmente de un país a otro. Por ejemplo, en España el límite se establece en los tres años, en Francia en los seis, y en Irlanda y Canadá en los dieciséis.

Asimismo, a pesar de que la tendencia general es no imponer condiciones de acceso a las familias (más allá de la edad de elegibilidad de los hijos/as), algunos países establecen una serie de restricciones tales como la obligación de realizar una actividad laboral (incluida la búsqueda activa de empleo) o formativa -Canadá, Estados Unidos-.

Otra importante constatación corresponde a la escasa presencia de criterios de renta a la hora de determinar el acceso y/o de definir el nivel de beneficio para las familias. Para definir dicho nivel de beneficios, una de las prácticas habituales es el establecimiento de un porcentaje sobre los gastos de cuidado, pese a que en algunos países se aplique a la totalidad. Además, todos los países imponen límites anuales, aunque estos pueden variar considerablemente.

Poniendo el foco sobre el crédito fiscal, una de las cuestiones centrales se refiere a su carácter reembolsable. Las deducciones fiscales reembolsables se diferencian de las deducciones tradicionales en la medida en que, si la deducción es superior a la deuda tributaria, la diferencia se abona a la persona contribuyente como prestación neta, como tramo negativo del impuesto. En todo caso, a diferencia de lo que ocurre en el caso de las prestaciones por hijo/a cargo, el uso de estas deducciones reembolsables es muy reducido en este tipo de prestaciones y únicamente Irlanda y Corea del Sur utilizan mecanismos de este tipo. 


\section{Referencias bibliográficas}

ADAMSON, P. (2008): “El cuidado infantil en los países industrializados: transición y cambio. Una tabla clasificatoria de la educación y los cuidados durante la primera infancia en los países económicamente avanzados", Innocenti Report Card, no 8, «https://www.unicef-irc. org/publications/514-el-cuidado-infantilen-los-pa\% $3 \%$ ADses-industrializadostransici\% $3 \%$ B $3 n-y$-cambio-una-tabla.html>.

ADEMA, W.; ALI, N. y THÉVENON, O. (2014): “Changes in family policies and outcomes: Is there convergence?", OECD Social, Employment and

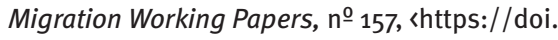
org/10.1787/5jz13wllxgzt-en〉.

ADO2, CONSULTORÍA, INVESTIGACIÓN Y COACHING (2014): Políticas de apoyo a las familias en Euskadi: análisis y propuestas, Vitoria-Gasteiz, Ararteko.

AHN, N. y MIRA, P. (2002): “A note on the changing relationship between fertility and female employment rates in developed countries", Journal of Population Economics, vol. 15, no 4, pp. 667-682, <https://doi.org/10.1007/ s001480100078>.

BECKER, G. (1981): A Treatise on the Family, National Bureau of Economic Research.

BOECKMANN, I.; MISRA, J. y BUDIG, M.J. (2015): "Cultural and institutional factors shaping mothers' employment and working hours in postindustrial countries", Social Forces, vol. 93, no 4, pp. 1.301-1.333, 〈https://doi.org/10.1093/ sf/sou119>.

BROOKS-GUNN, J.; HAN, W.J. y WALDFOGEL, J. (2002): "Maternal employment and child cognitive outcomes in the first three years of life: The NICHD Study of Early Child Care", Child Development, vol. 73, n- 4, pp. 1.052-1.072.
CARNEGIE TASK FORCE ON MEETING THE NEEDS OF YOUNG CHILDREN (1994): Starting Points: Meeting the Needs of Our Youngest Children, Carnegie Corporation New York.

CARNEIRO, P.; LØKEN, K. y SALVANES, K.G. (2015): “A flying start? Maternity leave benefits and long-run outcomes of children", Journal of Political Economy, vol. 123, nำ 2, pp. 365-412.

CASCIO, E.U. y WHITMORE SCHANZENBACH, D. (2013): “The impacts of expanding access to high-quality preschool education", Brookings Papers on Economic Activity, fall, pp. 127-178, «https:// doi.org/10.1353/eca.2013.0012〉.

CORNELISSEN, T.; DUSTMANN, C.; RAUTE, A. y SCHOENBERG, U. (2018): "Who benefits from universal child care? Estimating marginal returns to early child care attendance", European Economics: Labor \& Social Conditions eJournal, vol. 126, nํㅜ 6, pp. 2.356-2.409.

CUKROWSKA-TORZEWSKA, E. (2017): “Cross-country evidence on motherhood employment and wage gaps: The role of work-family policies and their interaction", Social Politics: International Studies in Gender, State \& Society, n-24, pp. 178-220, 〈https://doi.org/10.1093/sp/jxx004〉.

DAHL, G.B.; LøKEN, K.V.; MOGSTAD, M. y SALVANES, K.V. (2016): "What is the case for paid maternity leave?", The Review of Economics and Statistics, vol. 98, n- 4, pp. 655-670, 〈https:// doi.org/10.1162/REST_a_00602>.

DATTA GUPTA, N. (2018): “Maternity leave versus early childcare - What are the long-term consequences for children?", IZA World of Labor.

DATTA GUPTA, N. y SIMONSEN, M. (2016): “Academic performance and type of early childhood care", 
Economics of Education Review, vol. 53, C, pp. 217-229.

DATTA GUPTA, N.; SMITH, N. y VERNER, M. (2008): “The impact of Nordic countries' family friendly policies on employment, wages, and children", Review of Economics of the Household, $\mathrm{n} 06$, pp. 65-89.

DUSTMANN, C. y SCHÖNBERG, U. (2012): “Expansions in maternity leave coverage and children's longterm outcomes", American Economic Journal: Applied Economics, vol. 4, nํㅜ 3, pp. 190-224, 〈https://doi.org/10.1257/app.4.3.190〉.

EMAKUNDE (1996): Guía de desarrollo de acciones positivas, Vitoria-Gasteiz, Emakunde.

ERMISCH, J.F. y FRANCESCONI, M. (2001): "Family structure and children's achievements", Journal of Population Economics, vol. 14, n- 2, pp. 249270.

ESPING-ANDERSEN, G. (2004): "Untying the gordian knot of social inheritance", Research in Social Stratification and Mobility, nํ2, 21, pp. 115-138.

(2009): Incomplete Revolution: Adapting Welfare States to Women's New Roles, Cambridge, Policy Press.

GELBACH, J. (2002): "Public schooling for young children and maternal labor supply", American Economic Review, no 92, pp. 307-322.

GENNETIAN, L.A.; CASTELLS, N. y MORRIS, P. (2010): "Meeting the basic needs of children: Does income matter?", Children and Youth Services Review, vol. 32, no9, pp. 1.138-1.148, 〈https:// doi.org/10.1016/j.childyouth.2010.03.004'.

HECKMAN, J.J. (2006): "Skill formation and the economics of investing in disadvantaged children", Science, vol. 312, n- 5.782, pp. 1.900-1.902, 〈https://doi. org/10.1126/science.1128898>.

HECKMAN, J. y CARNEIRO, P. (2003): “Human Capital Policy”, NBER Working Paper Series, 〈https://EconPapers.repec.org/ RePEc:nbr:nberwo:9495〉.

HECKMAN, J.; GRUNEWALD, R. y REYNOLDS, A. (2006): “The dollars and cents of investing early: Costbenefit analysis in early care and education", Zero to Three, no 26, pp. 10-17.

HECKMAN, J.; MOON, S.H.; PINTO, R.; SAVELYEV, P. y YAVITZ, A. (2010): "The rate of return to the HighScope Perry Preschool Program", Journal of Public Economics, vol. 94, nํㅜ1-2, pp. 114-128.

HERBST, C.M. (2017): “Universal child care, maternal employment, and children's long-run outcomes: Evidence from the US Lanham Act of 1940", Journal of Labor Economics, vol. 35, nํㅜ 2, pp. 519-564.

KECK, W. y SARACENO, C. (2013): "The impact of different social-policy frameworks on social inequalities among women in the European Union: The labour-market participation of mothers", Social Politics: International Studies in Gender, State \& Society, vol. 20, no 3 , pp. 297-328, 〈https:// doi.org/10.1093/sp/jxto05>.

KLUVE, J. y SCHMITZ, S. (2014): “Social norms and mothers' labor market attachment - The medium-run effects of parental benefits", Ruhr Economic Papers, 〈https://doi.org/10.4419/86788545〉.
LALIVE, R. (2009): "How does parental leave affect fertility and return to work? Evidence from two natural experiments", The Quarterly Journal of Economics, n- 124 , pp. 1.363-1.402.

LATTIMORE, R. y POBKE, C. (2008): "Recent trends in Australian fertility", Productivity Commission Staff Working Papers.

LEFEVBRE, P.; MERRIGAN, P. y VERSTRAETE, M. (2009): "Dynamic labour supply effects of childcare subsidies: Evidence from a Canadian natural experiment on low-fee universal child care", Labour Economics, nำ 16, pp. 490-502.

LEITNER, S. (2003): "Varieties of familialism: The caring function of the family in comparative perspective”, European Societies, $\mathrm{n}^{0}-5, \mathrm{pp}$. 353-375.

LOVE, J.; ELIASON KISKER, E.; ROSS, C.; SCHOCHET, P.Z.; BROOKS-GUNN, J.; PAULSELL, D.; BOLLER, K.; CONSTANTINE, J.; VOGEL, C.; SIDLE FULIGNI, A. y BRAD, C. (2002): Making a Difference in the Lives of Infants and Toddlers and Their Families: The Impacts of Early Head Start. Volume I: Final Technical Report, Washington D.C., U.S. Department of Health and Human Services.

MATHIEU, S. (2013): “Entre l'effet tempo et l'effet quantum: une analyse de l'effet des politiques familiales sur la fécondité dans les pays avancés", Sociologie et Sociétés, vol. 45, n- 1, pp. 255276, 〈https://doi.org/10.7202/1016403ar〉.

MEYERS, M.K. (2003): Inequality in Early Childhood Education and Care: What Do We Know, Russell Sage Foundation.

NATIONAL RESEARCH COUNCIL E INSTITUTE OF MEDICINE (2003): Working Families and Growing Kids: Caring for Children and Adolescents, Washington D.C., The National Academies Press, 〈https://doi.org/10.17226/10669〉.

OLIVETTI, C. y PETRONGOLO, B. (2017): “The economic consequences of family policies: Lessons from a century of legislation in high-income countries", Journal of Economic Perspectives, $\mathrm{n}$ 31, pp. 205-230.

RAUTE, A. (2019): "Can financial incentives reduce the baby gap? Evidence from a reform in maternity leave benefits", Journal of Public Economics, vol. 169, 2019, pp. 203-222, 〈https://doi.org/10.1016/j. jpubec0.2018.07.010〉.

RUHM, C.J. (2000): "Parental leave and child health", Journal of Health Economics, vol. 19, no 6, pp. 931-96o, <https://doi.org/10.1016/s01676296(00)00047-3).

- (2004): "Parental employment and child cognitive development", The Journal of Human Resources, vol. 39, no 1, pp. 155-192, 〈https:// doi.org/10.2307/3559009>.

- (1998): "The economic consequences of parental leave mandates: Lessons from Europe", The Quarterly Journal of Economics, no 113, pp. 285-317.

SHIM, J. (2014): “Family leave policy and fertility rates in OECD countries including East Asia", en VV.AA., Population Association of America 2014 Annual Meeting, Population Association of America, 〈https://paa2014.princeton.edu/ abstracts/140973>. 
SHONKOFF, J.P. y PHILLIPS, D.A. (eds.) (2000): From Neurons to Neighborhoods: The Science of Early Childhood Development, Washington D.C., National Academies Press, <https://doi. org/10.17226/9824\%.

SIMONSEN, M. (2006): “Availability and price of high quality day care and female employment", Aarhus University Economics Papers.

THÉVENON, O. y GAUTHIER, A.H. (2011): "Family policies in developed countries: A 'fertility-booster' with side-effects", Community, Work \& Family, vol. 14, no 2, pp. 197-216, 〈https://doi.org/10.1080/ 13668803.2011.571400〉.

THÉVENON, O. y SOLAZ, A. (2013): “Labour market effects of parental leave policies in OECD countries", OECD Social, Employment and Migration Working Papers, 〈https://doi. org/10.1787/1815199X>.
WALDFOGEL, J. (1998): “Understanding the 'family gap' in pay for women with children", Journal of Economic Perspectives, vol. 12, nํㅜㄴ, pp. 137-156, 〈https://doi.org/10.1257/jep.12.1.137〉.

- (2004): "Social mobility, life chances, and the early years", CASE Papers, $\mathrm{n} \div 88$.

WASHBROOK, E.; RUHM, C.J.; WALDFOGEL, J. y HAN, W.J. (2011): "Public policies, women's employment after childbearing, and child well-being", Be Journal of Economic Analysis and Policy, 〈https://doi.org/10.2202/1935-1682.2938〉.

ZALAKAIN, J. (2021): “Diseño e impacto de las prestaciones por hijos a cargo en los países de la OCDE", Zerbitzuan, no 74, págs. \#\#-\#\#, 〈https://doi. org/10.5569/1134-7147.74.\#\#>. 